Cahiers de la recherche sur les droits

Cahiers

Fur les Droits fondamentaux

3 | 2004

Surveiller et punir / Surveiller ou punir ?

\title{
La protection du détenu par l'action du Comité européen pour la prévention de la torture
}

Jean-Manuel Larralde

\section{(2) OpenEdition}

Édition électronique

URL : https://journals.openedition.org/crdf/7466

DOI : $10.4000 /$ crdf.7466

ISSN : 2264-1246

Éditeur

Presses universitaires de Caen

Édition imprimée

Date de publication : 1 octobre 2004

Pagination : 29-42

ISBN : 2-84133-221-7

ISSN : $1634-8842$

Référence électronique

Jean-Manuel Larralde, « La protection du détenu par l'action du Comité européen pour la prévention de la torture », Cahiers de la recherche sur les droits fondamentaux [En ligne], 3 | 2004, mis en ligne le 18

décembre 2020, consulté le 18 novembre 2022. URL : http://journals.openedition.org/crdf/7466 ; DOI https://doi.org/10.4000/crdf.7466 


\title{
La protection du détenu par l'action du Comité européen pour la prévention de la torture
}

\author{
Jean-Manuel LARRALDE \\ Maître de conférences à I'Université de Caen Basse-Normandie
}

I. Un système préventif de protection des personnes incarcérées

A. Le CPT entre respect de la souveraineté étatique et autonomie d'action

B. Un rouage indispensable à la protection des personnes incarcérées par le Conseil de l'Europe

II. Un double apport à l'amélioration des conditions d'incarcération
A. Un « aiguillon " pour l'évolution des systèmes pénitentiaires nationaux
B. Une source d'inspiration pour la protection internationale contre la torture et les peines ou traitements inhumains et dégradants

\begin{abstract}
De manière assez compréhensible, la prison est souvent présentée comme un système clos, isolé du monde extérieur et peu perméable aux évolutions de la société. Or, si ce système " totalitaire ${ }^{1}$ s'analyse comme un instrument de punition et de réhabilitation, où les contraintes de sécurité sont prépondérantes, il constitue aussi une organisation relativement perméable, en raison notamment de l'existence d'une multiplicité de droits de regard tant internes qu'externes. La liste des différents contrôles, visites et inspections pouvant se dérouler dans les prisons françaises est en effet extrêmement impressionnante. Devraient se croiser en prison différentes structures et autorités permettant toutes, à leur niveau, d'assurer la transparence et l'efficacité de l'action pénitentiaire, et le respect des libertés individuelles. Le juge judiciaire a évidemment vocation à exercer une partie de ses missions en prison ${ }^{2}$. Plus précisément, le juge d'application des
\end{abstract}

peines, s'il ne peut «se substituer au directeur régional ou au chef de l'établissement, en ce qui concerne l'organisation ou le fonctionnement de celui-ci», doit néanmoins «assurer l'individualisation de l'exécution de la sentence judiciaire en orientant et en contrôlant les conditions de son application » (art. D 116 du Code de procédure pénale). La lecture du Code de procédure pénale permet même de découvrir une section intitulée «Du contrôle des établissements pénitentiaires ». L'article D 229 prévoit que «les établissements pénitentiaires font l'objet du contrôle général de l'inspection des services pénitentiaires et des inspections périodiques des magistrats ou des fonctionnaires de la direction de l'Administration pénitentiaire et des directeurs régionaux ou de leurs adjoints; en outre, ils sont soumis aux inspections du préfet ou du sous-préfet, ainsi que, dans le domaine de leur compétence, de toutes autres autorités administratives

1. Au sens d'E. Goffmann, Asiles, Paris, Minuit, 1968, p. 47; et de M. Foucault, Surveiller et Punir : naissance de la prison, Paris, Gallimard, 1975.

2. Même si l'article 727 , alinéa $1^{\text {er }}$, du Code de procédure pénale ne prévoit, pour le juge de l'application des peines, le juge d'instruction, le président de la chambre d'accusation, le procureur de la République et le procureur général, que le pouvoir de «visiter » les établissements pénitentiaires sans en préciser le contenu. Seul le juge de l'application des peines a l'obligation de visiter ces établissements au moins une fois par mois, afin de vérifier les conditions dans lesquelles les condamnés y exécutent leur peine (art. D 176). 
investies d'un pouvoir de contrôle à l'égard des différents services de l'Administration pénitentiaire». Bien plus encore, existe dans chacun des établissements, depuis le Premier Empire, une commission de surveillance (art. D 180 à D 185) se réunissant tous les ans et chargée « de la surveillance intérieure de l'établissement pénitentiaire en ce qui concerne la salubrité, la sécurité, le régime alimentaire et l'organisation des soins, le travail, la discipline et l'observation des règlements, ainsi que l'enseignement et la réinsertion sociale des détenus [...]»(art. D 184). Une volonté d'exhaustivité amène également à citer des inspections menées par les services des affaires sanitaires et sociales, l'Inspection du travail, l'Inspection du trésor, sans oublier l'éventuelle saisine du Médiateur de la République... La loi du 15 juin 2000 sur la présomption d'innocence a même récemment prévu que "Les députés et les sénateurs sont autorisés à visiter à tout moment les locaux de garde à vue, les centres de rétention, les zones d'attente et les établissements pénitentiaires » (art.720-1 A). Cette impressionnante liste semble donc faire de la France un État particulièrement respectueux des exigences tant internationales qu'européennes qui posent le principe des contrôles des établissements pénitentiaires ${ }^{3}$.

La réalité est tout autre et les différentes institutions qui se sont penchées depuis quelques années sur le monde carcéral ${ }^{4}$ ont toutes abouti à un constat identique: ces contrôles ne fonctionnent pas, étant pour la plupart d'entre eux soit inadéquats, soit totalement virtuels ${ }^{5}$. Comme l'indique le rapport de l'Assemblée nationale de 2000, "la multiplicité des contrôles ne signifie pas nécessairement qu'il y ait efficacité». Bien au contraire, ils paraissent «trop disséminés, parcellaires, pour constituer une véritable force de contrainte sur l'Administration pénitentiaire ${ }^{6}$. Face à ce large constat d'échec, une institution semble toutefois présenter un intérêt sur lequel tout le monde s'accorde. Il ne s'agit pas d'un contrôle interne, mais bien supranational et, qui plus est, récent car n'existant que depuis janvier 1989. Le Comité européen pour la prévention de la torture et des peines ou traitements inhumains ou dégradants (CPT), organe indépendant, est habilité à procéder à des visites inopinées des lieux de contrainte et de détention dans tous les
États membres du Conseil de l'Europe. Même si l'on ne peut évidemment faire reposer l'ensemble des contrôles sur cette seule institution, il n'en reste pas moins que ce Comité a réussi à s'imposer en un peu plus d'une décennie comme l'une des références du contrôle. Cette structure novatrice a pu développer un système cohérent et préventif de protection des personnes incarcérées, en réussissant tout à la fois à établir un équilibre entre respect de la souveraineté étatique et effectivité du contrôle et à trouver sa place dans la «politique pénitentiaire du Conseil de l'Europe» (I). Bien plus encore, le CPT apparaît aujourd'hui comme un élément d'orientation et de référence, pouvant peser sur les normes et pratiques pénitentiaires nationales et favoriser l'émergence d'un système effectif de contrôle au plan international (II).

\section{Un système préventif de protection des personnes incarcérées}

L'une des clés d'explication de la réussite du CPT réside dans l'architecture générale du dispositif instauré par la Convention de 1987. En effet, le Comité se révèle être un organe ayant trouvé un compromis entre le respect de la souveraineté des États visités et l'efficacité du contrôle (A). En outre, l'existence de cette institution a rendu plus cohérente la protection des personnes privées de liberté opérée par le Conseil de l'Europe en permettant des interactions réussies avec la jurisprudence de la Cour européenne des droits de l'homme (B).

\section{A. Le CPT entre respect de la souveraineté étatique et autonomie d'action}

C'est la Convention européenne pour la prévention de la torture et des peines ou traitements inhumains ou dégradants du 26 novembre 1987 qui instaure le CPT, qui constitue d'ailleurs l'objet principal de la Convention. En effet, selon l'article $1^{\mathrm{er}}$, « Par le moyen de visites, le Comité examine le traitement des personnes privées de liberté en vue de renforcer, le cas échéant, leur protection contre la torture et les peines ou traitements inhumains ou dégradants ». On chercherait en vain dans la Convention une

3. Article 55 de l'Ensemble de « règles minima » pour le traitement des détenus (résolution 663 C (XXIV) du 31 juillet 1957) : « Des inspecteurs qualifiés et expérimentés, nommés par une autorité compétente, devront procéder à l'inspection régulière des établissements et services pénitentiaires. Ils veilleront en particulier à ce que ces établissements soient administrés conformément aux lois et règlements en vigueur et dans le but d'atteindre les objectifs des services pénitentiaires et correctionnels »; Règle $n^{\circ} 4$ des Règles pénitentiaires européennes (Recommandation R (89) 3 du Comité des ministres du Conseil de l'Europe) : «Des inspecteurs qualifiés et expérimentés, nommés par une autorité compétente, devront procéder à l'inspection régulière des établissements et services pénitentiaires. Leur tâche consistera en particulier à veiller si et dans quelle mesure ces établissements sont administrés conformément aux lois et règlements en vigueur, aux objectifs des services pénitentiaires et aux normes imposées par les présentes règles. "

4. En particulier les deux missions d'enquête parlementaires sur les prisons de 2000 : rapport de M. J. Floch sur la situation dans les prisons françaises, Assemblée nationale, rapport 2251, 28 juin 2000, 2 tomes, et rapport de M. G.-P. Cabanel, Prisons: une humiliation pour la République, rapport de la commission d'enquête du Sénat, rapport 449, t. I, 28 juin 200o. Voir également G. Canivet, L'Amélioration du contrôle extérieur des établissements pénitentiaires, rapport remis à Madame le Garde des Sceaux, Ministre de la Justice, 6 juillet 2000 ; J.-M. Chauvet, La Sécurité des établissements pénitentiaires et des personnels, rapport à Madame le Garde des Sceaux, Ministre de la Justice, octobre 2001. Enfin, même s'il ne s'agit nullement d'un rapport officiel, il faut aussi rappeler l'ouvrage de V. Vasseur, Médecin-chef à la prison de la Santé, Paris, Le Cherche Midi, 2000.

5. Pour ne reprendre que cet exemple, voici comment M. Ivan Zakine, ancien membre du CPT, présentait devant la commisison d'enquête du Sénat le travail des commissions de surveillance: c'est une "grand-messe d'une solennité extrême. Quand cela se fait, on fait une visite au pas de course - parce qu'on n'a pas le temps - de ce que le chef d'établissement veut bien nous montrer: des locaux bien astiqués et bien propres. La visite se termine au mess des surveillants devant un apéritif. Le résultat n'est qu'un rituel qui n’a pas véritablement apporté quelque chose». Cf. G.-P. Cabanel, Prisons..., t. II, auditions.

6. Rapport de M. J. Floch sur la situation dans les prisons françaises, t. I. 
définition des pratiques prohibées mentionnées à l'article $1^{\text {er }}$. La Convention ne contient ni dispositions de fond sur la question de la torture et des peines ou traitements inhumains ou dégradants, ni tentatives de définition de ces notions. Seul est exprimé le postulat général de prohiber tout acte pouvant s'apparenter à un acte de torture ou traitement inhumain ou dégradant, que ces pratiques émanent de l'autorité publique, ou des personnes privées ellesmêmes sur un ou plusieurs de leurs camarades de détention.

La Convention de 1987, aujourd'hui ratifiée par les 45 États membres du Conseil de l'Europe, instaure un mécanisme non judiciaire, à caractère préventif, pour protéger toute personne privée de sa liberté. Ce mécanisme repose sur un système de visites effectuées par le CPT. Il comprend « un nombre de membres égal à celui des Parties» qui, à raison d'un membre par État, sont choisis «parmi des personnalités de haute moralité, connues pour leur compétence en matière de droits de l'homme ou ayant une expérience professionnelle dans les domaines dont traite la présente Convention» (art. 4 de la Convention). Les membres du CPT sont élus pour 4 ans par le Comité des ministres du Conseil de l'Europe, et peuvent être réélus deux fois. Il s'agit d'experts indépendants et impartiaux, regroupant tout à la fois juristes, médecins, psychiatres, spécialistes des questions pénitentiaires ou de la police. L'organe de contrôle est donc dès le départ conçu comme devant embrasser un domaine non strictement juridique, mais largement interdisciplinaire afin d'aborder la réalité complexe des lieux de détention.

Ce CPT se voit assigner par la Convention de 1987 un très vaste mandat, puisqu'il lui appartient d'établir si, en des lieux où des personnes sont privées de liberté par une autorité publique, il existe des conditions ou des circonstances, générales ou particulières, susceptibles de se dégrader au point de conduire à des actes de torture ou à des peines ou traitements inhumains ou dégradants, ou qui seraient en tout cas propices à la perpétration de tels actes ou pratiques inadmissibles. Le champ d'intervention du Comité n'est donc pas strictement pénitentiaire, car celuici voit sa compétence étendue à « tout lieu relevant de sa juridiction où des personnes sont privées de liberté par une autorité publique» (art. 2 de la Convention). Le CPT visite prisons et centres de détention pour mineurs, postes de police, centres de rétention pour étrangers, hôpitaux psychiatriques, afin d'évaluer la manière dont les personnes privées de liberté sont traitées et, le cas échéant, de recommander aux États des améliorations. Échappent cependant à la compétence du Comité que les « représentants ou délégués de puissances protectrices ou du Comité international de la Croix-Rouge visitent effectivement et régulièrement en vertu des Conventions de Genève du 12 août 1949 et de leurs Protocoles additionnels du 8 juin 1977» (art.17.3 de la Convention). Pour ne se limiter qu'aux personnes détenues dans les établissements pénitentiaires, le CPT était compétent, fin 2000, pour visiter 1867000 détenus $^{7}$.
Les visites sont effectuées par des délégations composées d'au moins deux membres du CPT, accompagnées de membres du Secrétariat du Comité et, si nécessaire, d'experts et d'interprètes. Le membre élu au titre du pays visité ne fait pas partie de la délégation en question.

Le CPT effectue des visites périodiques dans les États contractants ainsi que des visites « exigées par les circonstances» (dites visites ad hoc) selon l'article 7 de la Convention. Chaque année, il établit une liste de pays qui feront l'objet d'un programme de visites (dites visites périodiques) et qui concernent chaque État membre de manière régulière. L'État se voit notifier cette visite deux semaines avant son déroulement (le Comité étant tenu de notifier à l'État concerné son intention d'effectuer une visite en vertu de l'article 8 de la Convention). Mais la liste des lieux (qui n'est pas forcément exhaustive) n'est communiquée à l'État que quelques jours avant l'arrivée de la mission. Ces délais sont motivés par la volonté de laisser aux États la possibilité de préparer efficacement la visite, tout en ne leur laissant pas un temps suffisant pour masquer des éléments répréhensibles. En dehors des ces visites régulières d'une durée de une à deux semaines, le CPT peut également procéder à des visites ad hoc, d'une durée plus variable, en raison notamment d'allégations de faits de torture ou d'abus manifestes transmises au Comité. Enfin, il procède aussi à des visites de suivi, servant à s'assurer que les recommandations d'une précédente visite ont été suivies d'effet.

En 2002, le CPT a organisé 20 visites représentant une durée totale de 168 jours, divisées en 12 visites périodiques (122 jours) et 8 visites ad hoc (46 jours). Comme l'a clairement indiqué le CPT dans son Douzième rapport général d'activités, faute de renforcement supplémentaire du Secrétariat du Comité, il s'est vu contraint de limiter le programme des visites en 2002, comme en 2003, à un maximum de 170 jours. L'objectif du CPT pour 2004 est de tendre vers l'objectif de 200 jours de visite par an, volume requis pour faire face efficacement à la charge de travail engendrée par 45 Parties à la Convention ${ }^{8}$.

Le CPT constitue certes un organe de contrôle et d'inspection supranational, devant à ce titre respecter la souveraineté des États parties, mais jouissant néanmoins d'une latitude d'action considérable (voir l'article 8 de la Convention). En effet, il bénéficie notamment d'un accès au territoire de l'État concerné et le droit de s'y déplacer sans restrictions ; la possibilité de se rendre à son gré dans tout lieu où se trouvent des personnes privées de liberté, y compris le droit de se déplacer sans entrave à l'intérieur de ces lieux; l'accès à des renseignements complets sur les lieux où se trouvent des personnes privées de liberté ainsi qu'à toute autre information dont dispose la Partie et qui est nécessaire au Comité pour l'accomplissement de sa tâche; la possibilité de s'entretenir sans témoin avec les personnes privées de liberté. Seule la volonté clairement exprimée d'une personne privée de sa liberté de ne pas s'entretenir avec les membres du Comité pourrait être de 
nature à empêcher une audition (art. 8.3 de la Convention). En outre, les États ne peuvent formuler que des «objections » à l'occasion d'une visite. Très encadrées par l'article 9 de la Convention, de telles objections ne peuvent être faites que pour des motifs de défense nationale ou de sûreté publique ou en raison de troubles graves dans les lieux où des personnes sont privées de liberté, de l'état de santé d'une personne, d'un interrogatoire urgent, d'une enquête en cours en relation avec une infraction pénale grave.

Lors de ses visites, qui peuvent se dérouler de jour comme de nuit, le CPT doit examiner les conditions dans lesquelles sont détenues les personnes visitées, ce qui implique tout à la fois des entretiens avec les personnes privées de leur liberté (entretiens pouvant même aller jusqu'à recevoir des doléances de ces personnes), les autorités nationales et les responsables des établissements visités, mais également la vérification matérielle et générale des conditions de détention et même le contrôle des personnels concernés dans les différents établissements visités. Ces faits collectés aboutissent à un rapport adressé à l'État concerné. Ce rapport contient à la fois les faits, les appréciations du Comité et ses observations. Si nécessaire, le CPT recommande même des mesures concrètes afin de prévenir des situations qui pourraient aboutir à des actes de torture ou de peines ou traitements inhumains ou dégradants. Ce rapport constitue le point de départ d'un dialogue instauré avec l'État, qui dispose normalement d'un délai de six mois, à partir de sa communication, pour produire un rapport intérimaire en réponse, et d'un an pour fournir un rapport de suivi précisant les mesures prises pour apporter une solution aux dysfonctionnements constatés?.

Partant de l'idée que " la vie en prison est un tout " ${ }^{10}$, le contrôle opéré par le CPT est un contrôle global, s'attachant à vérifier tout ce qui relève des conditions de détention. Sont ainsi examinées les conditions générales de détention dans les établissements visités, ce qui comprend tout à la fois les locaux (espace dont disposent les détenus, éclairage, ventilation, installations sanitaires, repas et literie...) et les conditions sociales (relations avec les autres détenus et le personnel pénitentiaire, liens avec la famille ou les proches, avec les travailleurs sociaux et avec l'extérieur en général...), les soins de santé, les possibilités et conditions de travail. Le régime juridique de la détention (existence ou non des garanties fondamentales contre les mauvais traitements, notamment possibilité de déposer une plainte pour ces mauvais traitements ou mauvaises conditions de détention...) fait également l'objet d'une attention soutenue, comme les mesures pri- ses à l'égard des populations considérées comme à risques (mineurs, femmes, étrangers, toxicomanes...). Au-delà même des détenus, les personnels sont l'objet d'études minutieuses et ce tant en ce qui concerne leur formation que leur attitude ou leurs conditions de travail. La qualification de traitement inhumain ou dégradant, à laquelle peut conclure le CPT, résulte soit d'un élément spécifique, soit d'une combinaison de facteurs tels que la surpopulation, l'absence d'installations sanitaires et un régime alimentaire insuffisant. Le CPT doit non seulement vérifier si des abus sont effectivement commis mais aussi être attentif aux « indicateurs » ou "signes avant-coureurs » d'éventuels abus futurs.

La Convention de 1987 est parvenue à mettre en place un système efficace de contrôle tout en respectant la souveraineté étatique dans un domaine sensible, celui du droit pénal et des politiques pénitentiaires. Le travail du CPT repose sur une recherche de coopération maximum avec les États membres ${ }^{11}$. Selon l'article 10.1 de la Convention, toute visite du Comité aboutit à un rapport, qui doit relever les faits constatés et d'éventuelles recommandations, mais qui doit également tenir compte « de toutes observations éventuellement présentées par la Partie concernée ». Très fréquemment, le Comité, au sein même du rapport adressé au gouvernement du pays visité, demande à celui-ci de faire à son tour rapport, dans un délai déterminé, sur les mesures qu'il aura adoptées pour mettre en œuvre les recommandations formulées. L'État doit alors normalement rendre compte non seulement des mesures législatives et administratives qu'il aura, le cas échéant, été amené à prendre, mais aussi de l'application effective, dans la pratique, des recommandations du Comité. Par ses recommandations, le CPT a le pouvoir de proposer des mesures concrètes qu'il conviendrait de prendre immédiatement ou à long terme, de nature à mettre un terme aux errements constatés et à en prévenir d'autres, ou encore des normes plus générales que les États membres pourraient adopter en matière de détention. Seule l'absence de collaboration avec l'État concerné pourra motiver l'adoption (décidée à la majorité des deux tiers des membres du CPT) d'une déclaration publique ${ }^{12}$. Tous ces rapports sont toutefois normalement confidentiels. La confidentialité du rapport peut être écartée, mais il s'agit là d'une démarche explicite et volontaire de l'État concerné. Seul l'État peut demander la publication du rapport du Comité, ainsi que ses commentaires. Le respect du principe de confidentialité, mentionné par l'article 11 de la Convention, constitue d'ailleurs un élément majeur du travail du Comité ${ }^{13}$. Il signifie tout à la fois que

9. Ce formalisme procédural semble toutefois progressivement abandonné par le Comité, qui, le plus souvent, n'exige qu'un rapport de suivi étatique à l'issue de la première visite périodique, rapport suivi par une seule réponse lors des visites périodiques suivantes.

10. Voir G. Canivet, L'Amélioration. .

11. Néanmoins, le Comité peut communiquer aux autorités compétentes des «observations immédiates » dès la visite et ce dès que l'urgence de la situation le justifie (art. $8 \$ 5$ de la Convention).

12. Technique dont le CPT n'a pour l'instant fait qu'un usage très modéré avec seulement quatre déclarations publiques, deux à propos de la Turquie (1992 et 1996) et deux autres à propos de la situation dans la République russe de Tchétchénie (2001 et 2003).

13. Avec des exceptions parfois fâcheuses: ainsi, c'est dès le mois de janvier 2004 que l'Agence France Presse, relayée par plusieurs quotidiens nationaux, a révélé le contenu du rapport d'une visite ad hoc du Comité dans trois établissements pénitentiaires en juin 2003. Voir N. Guibert, «Surpeuplement, suicides, insalubrité en prison : l'accablant constat du Comité de prévention de la torture», Le Monde, 29 janvier 2004. 
le Comité se réunit à huis clos, que ses membres et ses collaborateurs sont tenus au secret professionnel, mais surtout que ses relations avec l'État, comme ses rapports, sont strictement confidentiels. Cette confidentialité constitue une règle intransgressible dans le travail du Comité. Il s'agit, en effet, de la contrepartie indispensable à un droit d'accès libre et sans entraves aux personnes privées de leur liberté.

Organe de dialogue avec les États, le CPT offre également l'image d'une coopération réussie à l'intérieur même des dispositifs établis depuis maintenant plusieurs décennies par le Conseil de l’Europe.

\section{B. Un rouage indispensable à la protection des personnes incarcérées par le Conseil de l’Europe}

La réussite du CPT apparaît aujourd'hui indéniable tant cette structure nouvelle a su rapidement s'imposer et démontrer l'intérêt de son action. Ce bilan positif est bien évidemment dû au travail du CPT et au contenu des inspections menées depuis maintenant près de quinze ans: le 27 janvier 2004, le Comité avait, depuis sa création, procédé à 170 visites ( 108 visites périodiques et 62 visites $a d$ hoc) et 120 rapports avaient été rendus publics. Mais cette réussite doit aussi s'analyser dans le cadre plus large du Conseil de l'Europe, dont le CPT ne constitue qu’un rouage. Comme il le définit lui-même, il est « avant tout et principalement un mécanisme de prévention des mauvais traitements bien qu'il puisse également, dans certains cas spécifiques, intervenir après que de tels faits aient eu lieu. En conséquence, alors que les activités de la Commission et de la Cour visent la "solution d'un conflit" au plan juridique, celles du CPT visent "l'évitement d'un conflit" au plan pratique ${ }^{14}$. Le CPT ne doit pas être analysé comme une structure isolée, mais bien comme un rouage spécifique du complexe dispositif de protection des droits de l'homme construit dans le cadre du Conseil de l'Europe «mettant en place un mécanisme non judiciaire "pro-actif" en parallèle au mécanisme judiciaire de contrôle a posteriori de la Cour européenne des Droits de l'Homme ${ }^{15}$.

Il s'agit tout d'abord d'un corps de règles contenues dans la Recommandation R (87) 3 du Conseil de l'Europe, instaurant les Règles pénitentiaires européennes. Elles ne constituent certes pas un instrument intrinsèquement contraignant, mais selon l'exposé des motifs «elles ont largement valeur de code de déontologie pour l'administration et le traitement pénitentiaires " ${ }^{16}$. C'est leur intégration dans les réglementations pénitentiaires nationales qui leur donne une force contraignante, mais leur existence même procure aux États membres des standards de référence, en deçà desquels le traitement de leurs détenus n'est plus conforme aux principes d'un État démocratique.
Le deuxième niveau de protection est constitué par un système de visites indépendantes, tendant à prévenir la survenance de faits constitutifs de torture ou de peines ou traitements inhumains ou dégradants. Il s'agit là de la tâche spécifiquement assignée au CPT. Sa tâche est de prévenir de mauvais traitements, physiques ou mentaux, à l'encontre des personnes privées de liberté. Son attention est plutôt tournée vers l'avenir que le passé.

Enfin, le troisième niveau de protection réside dans une possibilité de recours juridictionnel, avec la possibilité pour toute personne privée de sa liberté de saisir la Cour européenne des droits de l'homme. En effet, la Commission européenne des droits de l'homme a très tôt considéré que la détention d'une personne ne prive pas celle-ci «de la garantie des droits et libertés définis dans la Convention ${ }^{17}$. Même si, à la différence d'autres normes internationales, la Convention européenne ne contient pas de règles explicites relatives à la protection des personnes incarcérées, la Cour a réussi à imposer une jurisprudence dynamique et protectrice, en s'appuyant sur plusieurs dispositions de la Convention. Au cœur de la jurisprudence européenne figure la protection du détenu contre les actes de torture, les peines ou traitements inhumains et dégradants (art. 3 de la Convention). Cette disposition a d'ailleurs inspiré la rédaction en 1987 de la Convention européenne pour la prévention de la torture et des peines ou traitements inhumains ou dégradants et l'article 3 est rappelé dans le Préambule de la Convention de 1987. Le travail jurisprudentiel opéré par la Cour recherche un compromis raisonnable entre les nécessaires contraintes de la détention et le non moins nécessaire respect de la dignité du détenu. Une étude de l'évolution de la jurisprudence de l'article 3 appliqué aux détenus montre un renforcement progressif des exigences pesant sur les États en matière pénitentiaire. Initialement, s'agissant du régime de la détention, la Commission européenne des droits de l'homme exigeait un seuil de gravité assez élevé pour conclure à la violation de l'article 3 de la Convention. Par exemple, l'existence de conditions matérielles de détention déplorables n'était pas, en elle-même, contraire à l'article 3. La violation n'existait que lorsque ces conditions devenaient dégradantes en particulier en raison d'une absence totale d'hygiène ou de la saleté des cellules. Ainsi pour la Commission européenne des droits de l'homme, « des conditions de détention qui, sous certains aspects, seraient en retrait par rapport aux "Règles minima" ne constitueraient pas, de ce seul fait, un traitement inhumain ou dégradant $»$. Seules des conditions de détention constituant une violation directe de l'article 3 de la Convention (ce qui n'était pas le cas en l'espèce) pouvaient être condamnées par la Commission ${ }^{18}$. Cette approche semblant légitimer des conditions de détention inacceptables, voire couvrir certaines formes de violence

14. Premier rapport général d'activités du CPT couvrant la période du $1^{\mathrm{er}}$ novembre 1989 au 31 décembre 1990, CPT/ Inf (91) 3 [FR], 20 février 1991.

15. Voir, notamment, le Douzième rapport général d'activités du CPT, 2002, préface.

16. Sur ce point, voir notre étude «Le soft law européen comme promoteur des droits des personnes incarcérées", in Questions sur le droit européen,

C. Grewe (dir.), Caen, Presses universitaires de Caen, 1996, p. 189-208.

17. Requête $n^{\circ} 1670$ / 61, Ilse Koch c. RFA, Annuaire $n^{\circ}$ 5, p. 126.

18. Requête $n^{\circ} 7341 / 76$, Eggs c. Suisse, décision du 11 décembre 1976, Décisions et rapports $n^{\circ}$ 6, p. 176 . 
carcérale, n'est plus de mise aujourd'hui. En effet, la Cour européenne applique depuis quelques années plusieurs concepts protecteurs de la situation des personnes privées de leur liberté. Avec l'arrêt Kudla c. Pologne du 26 octobre 2000 apparaît pour la première fois le droit pour tout prisonnier à des conditions de détention humaines. Un tel droit implique que les modalités d'exécution de la peine de prison «ne soumettent pas l'intéressé à une détresse ou à une épreuve d'une intensité qui excède le niveau inévitable de souffrance inhérent à la détention et que, eu égard aux exigences pratiques de l'emprisonnement, la santé et le bien être du prisonnier soient assurés de manière adéquate, notamment par l'administration des soins médicaux ${ }^{19}$. Ceci implique notamment qu'une personne gravement handicapée doit bénéficier de conditions de détention adaptées à ses besoins spécifiques. Il revient même désormais à l'État de prendre préventivement des mesures nécessaires à la santé et à l'intégrité physique des détenus. Selon l'arrêt Pantea c. Roumanie du 3 juin 2003, les autorités internes, dès lors qu'elles sont au courant d'agissements contraires à la dignité du détenu, ont, dans le cadre de leur devoir de surveillance des personnes privées de liberté, l'obligation de prendre les mesures visant à empêcher la matérialisation de ce risque ${ }^{20}$.

Cette évolution de la jurisprudence de la Cour européenne des droits de l'homme démontre que Cour comme Comité participent à un même mouvement, tendant, d'une manière générale, au renforcement de la dignité des personnes incarcérées. Même si le CPT constitue un système indépendant de la Convention européenne des droits de l'homme, les interactions entre les deux structures et mécanismes sont évidentes. L'article 17.2 de la Convention de 1987 prévoit d'ailleurs une articulation avec le dispositif juridictionnel instauré par la Convention européenne des droits de l'homme: «Aucune disposition de la présente Convention ne peut être interprétée comme une limite ou une dérogation aux compétences des organes de la Convention européenne des Droits de l'Homme ou aux obligations assumées par les Parties en vertu de cette Convention. » Il est également assez clair que si le Comité conclut à des actes s'apparentant à des tortures ou à des peines ou traitements inhumains ou dégradants vis-à-vis d'un détenu, les personnes concernées risquent de saisir la Cour européenne des droits de l'homme afin de faire condamner l'État concerné pour violation de l'article 3 de la Convention. Ces rapports entre Cour et Comité s'articulent autour de deux mouvements partiellement contradictoires, établissant à la fois l'autonomie des deux systèmes et d'indéniables interactions.
Dans l'exercice de ses missions, «le CPT est en droit d'utiliser les normes juridiques contenues non seulement dans la Convention européenne des Droits de l'Homme, mais aussi dans un certain nombre d'autres instruments pertinents ayant trait aux droits de l'homme (ainsi que l'interprétation qui en est faite par les organes des droits de l'homme respectivement compétents). Le Comité n'est toutefois pas lié par la jurisprudence des organes judiciaires ou quasi-judiciaires agissant dans ce même domaine. Cette constatation vaut notamment pour la jurisprudence de la Cour européenne des droits de l'homme ${ }^{21}$.

Ceci explique tout d'abord que le Comité se serve de la jurisprudence de la Commission et de la Cour européennes des droits de l'homme comme d'un guide de référence et non comme d'une source indiscutable. Le CPT peut donc parfaitement s'écarter de la jurisprudence de la Cour européenne des droits de l'homme, en reconnaissant notamment des situations s'apparentant à des peines ou traitements inhumains ou dégradants, alors même que la Cour ne voit pas de violation de l'article 3 dans des espèces comparables. Le Comité explique luimême que ces normes « externes» (dont la jurisprudence de la Cour) ne sont que des «points de départ» ou des «références» "lors de l'évaluation du traitement des personnes privées de liberté dans les différents pays ${ }^{22}$. Ceci explique que le Comité s'est progressivement construit son propre corps de règles générales, destinées à servir de principes directeurs et d'éléments d'orientation pour les États membres. Ces règles, formalisées dans les Rapports généraux d'activités, font l'objet d'une compilation tenue à jour et sont connues sous le nom de «normes du CPT ${ }^{23}$.

Cette autonomie explique aussi que le Comité a progressivement développé des concepts autonomes de la «torture » et des peines ou traitements « inhumains» ou «dégradants» qui ne correspondent pas tout à fait aux définitions et méthodes de la Cour européenne des droits de l'homme. Cette dissociation conceptuelle s'explique en particulier parce que le CPT, pour s'acquitter avec efficacité de sa fonction de prévention, doit tendre vers un degré de protection plus élevé que celui que la Cour européenne des droits de l'homme retient lorsqu'elle se prononce dans des affaires concernant les mauvais traitements aux personnes privées de liberté et leurs conditions de détention. Cela signifie notamment que le CPT peut retenir le caractère de traitements inhumains et dégradants pour certaines conditions de détention marquées par le surpeuplement, le manque d'installations sanitaires et un régime de détention inapproprié, alors même que la Commission européenne des droits de

19. Note F. Sudre, «Droit de la Convention européenne des droits de l'homme», JCP, chronique I, 291, 2001, p. 128 sq.

20. Ibid., 160, 2003, p. 1570.

21. Premier rapport général d'activités, 1991, \$5, CPT / Inf (91) 3 (FR).

22. Ibid.

23. Doc. CPT / Inf / E (2003) 1. On trouve donc désormais des règles générales touchant à la détention par la police (Deuxième, sixième et douzième rapports généraux d'activités), à l'emprisonnement (Deuxième, septième et onzième rapports), à la formation des responsables de l'application des lois (Deuxième rapport), aux services de santé dans les prisons (Troisième rapport), aux personnes retenues en vertu de législations relatives à l'entrée et au séjour des étrangers (Septième rapport), à l'éloignement d'étrangers par la voie aérienne (Treizième rapport), au placement non volontaire en établissement psychiatrique (Huitième rapport), aux mineurs privés de liberté (Neuvième rapport), aux femmes privées de liberté (Dixième rapport). 
l'homme avait refusé d'admettre que de telles pratiques revêtaient une gravité susceptible d'atteindre le seuil de gravité permettant de reconnaître une violation de l'article 3 de la Convention européenne des droits de l'homme. La Cour européenne des droits de l'homme utilise une technique de « seuils » pour qualifier les actes prohibés de « torture », ou de peines ou traitements « inhumains » ou « dégradants ». Cela signifie donc qu'une même pratique à l'encontre d'un détenu peut potentiellement relever des trois qualificatifs, en fonction de sa gravité et de son intensité. La torture se définit ainsi pour la Cour comme une forme aggravée des autres mauvais traitements. Le CPT, pour sa part, semble avoir retenu une approche quelque peu différente : ainsi, la torture relèverait davantage de formes spéciales de violences employées à dessein (principalement par des formes de police) pour extorquer aveux ou informations, ou, plus généralement, pour intimider ou humilier des détenus ${ }^{24}$. Ce faisant, la Cour se situerait dans une perspective plus proche de l'article $1^{\text {er }}$ de la Convention onusienne de 1984 que de la définition habituelle de la torture dans la jurisprudence de la Cour européenne des droits de l'homme. Par contre, les peines ou traitements inhumains ou dégradants (utilisés spécialement ou de manière conjointe) relèveraient davantage d'aspects du régime de détention (surpeuplement carcéral combiné avec des locaux insalubres, isolement prolongé).

De son côté, la Cour européenne des droits de l'homme n'est pas liée par les rapports de visite du CPT. La Commission européenne des droits de l'homme avait ainsi rejeté des rapports invoqués par un requérant, en indiquant qu'il ne lui appartenait pas de se prononcer sur les conditions générales de détention ${ }^{25}$. Plus récemment, dans l'affaire Peers c. Grèce (19 avril 2001) la Cour constate que les délégués de la Commission européenne des droits de l'homme ne sont pas parvenus aux mêmes conclusions que le CPT en ce qui concerne les conditions de détention de la prison de Koridallos (principalement parce que les deux visites ne se sont pas déroulées dans les mêmes conditions climatiques). La Cour ne va alors se fonder que sur les faits établis par la Commission, en motivant pourquoi elle ne suit pas totalement les conclusions du CPT ${ }^{26}$.

Cette autonomie organique et conceptuelle relevée entre la Cour européenne des droits de l'homme et le CPT ne doit toutefois pas masquer l'existence de larges interactions.
Le CPT n'est en effet nullement étranger à l'évolution de la jurisprudence de la Cour européenne des droits de l'homme et il se tient évidemment informé de manière précise de toutes les évolutions jurisprudentielles. Ce travail de surveillance l'amène parfois à se féliciter lorsqu'il lui apparaît que la Cour souscrit à son analyse ${ }^{27}$. Le Comité est même allé jusqu'à faire observer que la Cour européenne des droits de l'homme constituait un mécanisme plus efficace que lui-même pour examiner des allégations émanant de personnes menacées d'expulsion et même «le cas échéant, prendre des mesures préventives ». La répartition des rôles entre un CPT «préventif » et une Cour intervenant a posteriori apparaît donc ici totalement inversée !

Si le CPT utilise indéniablement les matériaux fournis par la Cour européenne des droits de l'homme, l'interaction entre les deux systèmes se manifeste surtout dans une utilisation de plus en plus fréquente du travail du CPT par la Cour ${ }^{28}$. La Cour européenne des droits de l'homme doit toujours, afin de rendre son arrêt, établir les faits, moment dans la construction de l'arrêt qui comprend tout à la fois les "circonstances de l'espèce " (faits et déroulement de la procédure en droit interne), mais aussi « le droit et la pratique interne pertinents ». C'est à ce stade d'élaboration de l'arrêt que la Cour européenne a pris l'habitude depuis quelques années de s'appuyer, au-delà du droit et de la jurisprudence internes pertinents, sur les rapports du Comité. Cette technique faisant des travaux du Comité une source d'inspiration principale lorsque la requête concerne une personne privée de sa liberté se trouve notamment dans l'arrêt Aerts c. Belgique, rendu par la Cour le 30 juillet $1998^{29}$. La Cour se sert en effet du rapport rendu public par la Belgique en 1994 à l'occasion de la visite du CPT de 1993, plus spécifiquement en ce qui concerne les développement relatifs à l'annexe psychiatrique de la prison de Lantin ${ }^{30}$. La Cour mentionne également, dans un très long extrait, le rapport de suivi intérimaire rendu public par la Belgique en réponse au rapport du CPT (\$29). L’arrêt Kalachnikov c. la Russie du 15 juillet 2002 offre une autre utilisation intéressante des travaux du CPT par la Cour européenne des droits de l'homme. Devant déterminer si les conditions de détention du requérant dans une maison d'arrêt pouvaient entraîner une violation de l'article 3, la Cour va s'appuyer sur sa jurisprudence, mais également sur les exigences du $\mathrm{CPT}$ en la matière. Plus spécifiquement, elle va indiquer

24. Cette analyse étant notamment défendue par R. Morgan et M. Evans, Combattre la torture..., p. 65.

25. Com. EDH, Delazarus c. Royaume-Uni, requête n ${ }^{\circ} 19066$ / 91, Revue française de droit administratif, 1994, p. 1195 sq.

26. La Cour explique que parce que «les délégués ont procédé à des investigations approfondies sur les griefs du requérant, et ont accordé une attention particulière pendant leur inspection aux conditions prévalant à l'endroit même où l'intéressé avait été détenu ", elle estime " pouvoir se fonder sur les constatations des délégués de la Commission» ( $\$ 70$ de l’arrêt).

27. Ainsi dans son Septième rapport général d'activités, le Comité se félicite du jugement rendu le 25 juin 1996 par la Cour européenne des droits de l'homme dans l'affaire Amuur c. France, qui peut être considéré comme une confirmation du point de vue du Comité en indiquant qu'un séjour dans une zone de transit ou «internationale » peut, selon les circonstances, s'apparenter à une privation de liberté au sens de l'article 5 (1) (f) de la Convention européenne des droits de l'homme ( $\$ 26$ du rapport).

28. Et par les requérants: dans l'arrêt Van der Ven c. Pays-Bas du 4 février 2003, c'est le requérant qui considère qu'à la lumière des commentaires très critiques formulés par le CPT sur divers aspects du régime de l'EBI (établissement de sécurité maximale), il ne peut y avoir aucun doute que celui-ci doit être qualifié d'inhumain $(\$ 38)$.

29. Note J.-P. Céré, Dalloz, 2001, nº 29, p. 2336 sq.; E. Boitard, note, Les Petites Affiches, 20 septembre 2001, $n^{\circ}$ 188, p. 19 secondes; voir également Cour $\mathrm{EDH}, 15$ janvier 2004, Sakkopoulos c. Grèce.

30. Rapport au gouvernement de la Belgique relatif à la visite effectuée par le CPT en Belgique du 14 au 23 novembre 1993, CPT / Inf (94) 15. 
que «le Comité européen pour la prévention de la torture et des peines ou traitements inhumains ou dégradants a fixé à sept mètres carrés par personne la surface minimum approximative souhaitable pour une cellule de détention » (\$ 97 de l'arrêt). Or la Cour indique qu'alors que la cellule était conçue pour huit prisonniers, le nombre de détenus habituellement présents dans la cellule pendant toute la période où le requérant a été emprisonné a, selon les observations de celui-ci transmises à la Cour, varié entre dix-huit et vingt-quatre. Les conditions matérielles de détention en l'espèce ne semblent donc plus discutables à partir du moment où le Comité a donné son « interprétation officielle». Ceci conduit fort logiquement la Cour à déclarer qu' «à la lumière de ce qui précède, la Cour conclut que les conditions de détention du requérant, en particulier la surpopulation et l'insalubrité extrêmes, et leurs effets préjudiciables sur la santé et le bien-être de l'intéressé, combinées avec la durée de la période pendant laquelle il a été détenu dans de telles conditions, s'analysent en un traitement dégradant " et donc en une violation de l'article 3 de la Convention. Dans l'arrêt Mouisel c. France du 14 novembre $2002^{31}$, la Cour va même au-delà de l'établissement des faits par le Comité, en faisant des rapports du CPT une partie du «droit pertinent» applicable pour parvenir à la solution de l'affaire ${ }^{32}$. La Cour prend notamment acte des recommandations formulées par le CPT quant aux conditions de transfert et d'examen médical des détenus qui continuent, selon celui-ci, de poser problème au regard de l'éthique médicale et du respect de la dignité humaine. Cet arrêt Mouisel montre parfaitement le double intérêt des rapports du CPT dans la construction d'une jurisprudence européenne: raisonnement par analogie si l'établissement concerné par la requête a été visité par le Comité, mais également utilisation des conclusions générales publiées dans les rapports annuels du CPT. Ainsi la Cour dans cette espèce utilise le troisième rapport annuel du CPT en y extrayant les mentions relatives aux services de santé dans les prisons, où le Comité précise que, selon lui, la détention continue des détenus « qui présentent un pronostic fatal à court terme, [...] qui souffrent d'une affection grave dont le traitement ne peut être conduit correctement dans les conditions de la détention [ou] qui sont sévèrement handicapés ou d'un grand âge $[. .$.$] peut créer une$ situation humainement intolérable» ( $\$ 28$ de l'arrêt). Plus récemment, l'arrêt Yankov c. Bulgarie du 11 décembre 2003 utilise les recommandations générales du CPT qui avaient été formulées lors d'une visite en ex-République yougoslave de Macédoine demandant de mettre fin aux pratiques consistant à raser la tête des détenus nouveaux arrivants dans les établissements pénitentiaires ${ }^{33}$ pour faire de ce type de pratique un traitement ayant le caractère de traitement dégradant dans le cas d'espèce ${ }^{34}$.

\section{Un double apport à l'amélioration des conditions d'incarcération}

Même si l'apport direct du CPT s'avère parfois délicat à déterminer, il est incontestable que cette institution constitue l'un des éléments qui contribuent à l'amélioration des standards pénitentiaires, le Comité peut ici être comparé à un véritable "aiguillon » vis-à-vis des États (A). Mais l'intérêt du Comité a également dépassé les frontières géographiques de l'Europe, servant ainsi de modèle et de référence dans la construction d'un système de protection des droits des personnes privées de leur liberté au plan international (B).

\section{A. Un « aiguillon " pour l'évolution des systèmes pénitentiaires nationaux}

M. Ivan Zakine, ancien membre du CPT, a parfaitement défini la portée exacte du CPT: «Le Comité européen est là pour tirer les sonnettes d'alarme. Aux gouvernements à prendre le relais pour mettre en ouvre, aux organes nationaux, gouvernementaux ou non gouvernementaux, à rester vigilants » ${ }^{35}$. Le Comité n'est évidemment pas à même d'imposer des mesures aux États, de les contraindre à cesser certaines pratiques ou comportements, ni de prendre des normes à la place des organes nationaux compétents. L'apport du Comité s'analyse davantage en un travail de persuasion, de contrôles et d'échanges sur les États, qui finit par peser sur les systèmes pénitentiaires nationaux. Il reste néanmoins que l'influence exacte du CPT et sa participation exacte à l'évolution des systèmes nationaux sont éminemment variables et dépendent très largement de la bonne volonté des États concernés à «jouer le jeu » de la collaboration avec le Comité. Les États sont le plus souvent des partenaires loyaux du CPT et le premier signe d'acceptation du Comité réside dans le renversement du principe de confidentialité des rapports de visite posé par l'article 11 de la Convention: alors que cette disposition indique que « les informations recueillies par le Comité à l'occasion d'une visite, son rapport et ses consultations avec la Partie concernée sont confidentiels ", les chiffres fournis par le CPT montrent que les États ont pris l'habitude de publier rapports et commentaires comme l'alinéa 2 de cet article le leur permet. Au 27 janvier 2004, sur les 170 visites (108 visites périodiques et 62 visites ad hoc), 120 rapports ont été rendus publics ${ }^{36}$. Ce chiffre est révélateur

31. Note H. Moutouh, Dalloz, 2003, $\mathrm{n}^{\circ}$ 5, p. 303 sq.

32. Voir également Cour EDH, Matencio c. France, 15 janvier 2004, $\$ 34$ sq.

33. Report on the visit to the former Yugoslav Republic of Macedonia in 1998, 11 octobre 2001, $\$ 50$.

34. La volonté d’humilier le détenu ayant été, pour la Cour, la principale motivation de cette tonte forcée. Voir les $\$ 100 s q$. de l'arrêt.

35. Voir G.-P. Cabanel, Prisons..., t. II, auditions.

36. Selon les chiffres transmis par le CPT lui-même (http://www.cpt.coe.int/fr/apropos.htm). Matériellement, les États autorisent le plus souvent la publication du rapport en même temps que leur réponse, ce qui occasionne des délais de publication relativement longs allant de dix-huit mois à deux ans. Chaque rapport général d'activités du CPT comprend désormais un tableau récapitulant les visites par État et le nombre de rapports autorisés à la publication. 
d'un fort degré d'acceptation des travaux du Comité au sein des États membres. De manière assez peu surprenante, les différents rapports annuels du CPT expriment que le dialogue avec les États visités se poursuit « globalement de façon satisfaisante ${ }^{37}$. Les rapports intérimaires et de suivi demandés par le CPT sont transmis par les États concernés, en respectant généralement les limites de temps imposées. Certains de ces rapports apportent des réponses extrêmement précises et motivées et tous témoignent que les recommandations et autres conseils du CPT sont le plus souvent pris en considération ${ }^{38}$. Il n'en reste pas moins que l'on ne peut se contenter d'un satisfecit purement diplomatique et il s'avère plus délicat de déterminer concrètement le contenu du dialogue entre le Comité et les États membres ${ }^{39}$. En effet, aucun État partie n'a pour l'instant souhaité publier l'ensemble de ses communications avec le Comité ${ }^{40}$.

La portée des travaux du Comité est parfois directement visible, les États opérant certaines transformations ou modifications sous l'influence directe d'un rapport de visite ou à l'occasion même d'une visite. Ainsi en est-il de la fermeture de cellules collectives et de l'amélioration rapide des conditions matérielles de détention dans les cellules de sûreté des prisons de Berne et de Zurich, dont la prise en charge de certains détenus a été assurée par le service de garde d'un hôpital extérieur. C'est également en Suisse qu'à la suite d' " observations immédiates » formulées lors d'une visite du CPT, et qui considéraient certaines cellules comme insatisfaisantes, que les autorités ont fait savoir rapidement que ces cellules avaient été évacuées et que des travaux étaient en cours ${ }^{41}$. La même mesure a été prise par le gouvernement néerlandais en mettant hors service des locaux cellulaires le jour même de la visite d'une délégation du $\mathrm{CPT}^{42}$. De même, les observations relatives aux conditions matérielles de détention dans les prisons islandaises demandant la fermeture des cellules de moins de $5 \mathrm{~m}^{2}$ ont été suivies d'effets dans les quatre mois suivant la visite du CPT ${ }^{43}$. Plus récemment, c'est en réponse à la critique du $\mathrm{CPT}$ sur le temps hors cellule et les activités limités des personnes détenues en vertu de la loi de 2001 relative à la lutte contre le terrorisme, le crime et à la sécurité que les autorités du Royaume-Uni ont indiqué que les intéressés avaient été transférés dans des unités capables de leur proposer plus d'activités ${ }^{44}$. Une visite du CPT peut également aboutir à activer des mécanismes de plaintes. Ainsi en Grèce, c'est à la suite de recommandations du Comité qu'une inspection administrative a été diligentée dans un établissement pénitentiaire et a débouché sur des mesures concrètes consistant notamment dans le transfert de délinquants vers un autre établissement ${ }^{45}$. En France, c'est à l'occasion d'une visite d'une mission d'inspection du CPT en 1996 qu'une enquête administrative a pu se déclencher concernant des sévices sexuels infligés aux détenus. Suite à une visite au centre des jeunes détenus de Fleury-Mérogis, le CPT avait eu vent d'une enquête administrative discrète concernant un viol collectif dénoncé par un travesti. Le CPT avait alors alerté la Chancellerie qui avait saisi le Parquet d'Évry. L'affaire s'est terminée par la condamnation de trois surveillants de la prison de Fleury-Mérogis ${ }^{46}$. Ces conséquences restent toutefois exceptionnelles dans la pratique du Comité. En effet, sa visite correspond davantage au déclenchement d'un processus de dialogue et de concertation, qu'à une fin en soi.

Dans d'autres cas, les évolutions sont probablement dues à l'influence du Comité, même si dans ces hypothèses il ne constitue que l'un des éléments déclencheurs de la réforme. On peut à ce titre citer l'adoption par la France du décret du 2 avril 1996 relatif au régime disciplinaire des détenus. Ce texte porte notamment réforme de la procédure disciplinaire, en instaurant une commission de discipline. Parmi les éléments ayant incité la France à instaurer cette nouvelle structure au sein des établissements pénitentiaires figure, sans nul doute, la description très édifiante d'une procédure disciplinaire à la maison d'arrêt de Marseille-Baumettes ${ }^{47}$. En juin 2002, la présidente du CPT, $\mathrm{M}^{\mathrm{me}}$ Sylvia Casale, indiquait certains

37. Voir notamment le Troisième rapport général d'activités, 1993, CPT / Inf (93) 12 (FR), \$15.

38. L'utilisation du Rapport de réponse par l'État peut d'ailleurs représenter un intéressant instrument de publicité: tout en répondant aux critiques du Comité de manière précise, l'État en profite, dans le même temps, pour légitimer les nouvelles orientations de sa politique pénitentiaire. Voir, par exemple, le Rapport de suivi du gouvernement Belge de 1999, où le gouvernement utilise ce document pour présenter sa nouvelle loi sur la libération conditionnelle «plus respectueuse des droits des détenus».

39. Voir M.-J. Bernard, «Conseil de l'Europe, droits de l'homme et prison : l'impact du Comité européen pour la prévention de la torture sur la réforme des prisons ", Revue française d'administration publique, $\mathrm{n}^{\circ}$ 99, juillet-septembre 2001, p. 525 sq.

40. Le CPT fait d'ailleurs parfois son autocritique, en relevant "qu'il est loin d'être satisfait de ses propres performances en ce qui concerne le dialogue permanent ", principalement "en raison des problèmes liés à la charge de travail au sein de son Secrétariat ", Cinquième rapport général d'activités, $1995, \$ 10$.

41. A. Cassese, Inhuman States, Emprisonment, Detention and Torture in Europe Today, Cambridge, Polity Press, 1996, p. 41.

42. Rapport au gouvernement des Pays-Bas relatif à la visite effectuée par le CPT aux Pays-Bas du 30 août au 8 septembre 1992, 1993, CPT / Inf (93) 15 (FR), $\$ 25$.

43. Lettre de réponse des autorités islandaises aux observations immédiates du CPT en date du 27 décembre 1993 postérieurement à la visite de juillet 1993.

44. Douzième rapport général d'activités, 2003, \$10.

45. Rapport au gouvernement grec relatif à la visite effectuée par le CPT en Grèce du 14 au 26 mars 1993, CPT / Inf (93) 3 (FR), \$ 101 et 271.

46. E. Inciyan, «Trois surveillants de la prison de Fleury-Mérogis condamnés pour agressions sexuelles sur des travestis», Le Monde, 3 février 1999.

47. «La qualité des débats était tout sauf satisfaisante. La délégation a été particulièrement frappée par l'absence de sérénité qui présidait l'audience. En effet, en plus de l'autorité investie du droit de punir et du personnel strictement nécessaire au déroulement de la procédure (greffier/ secrétaire, etc.), s'ajoutait un groupe de surveillants "spectateurs", à l'intérieur et à l'extérieur de la salle. Ces derniers formulaient les commentaires qu'ils considéraient utiles au fur et à mesure du déroulement de l'audience. Cet effet de "chœur" engendrait une atmosphère vindicative tout à fait inappropriée», Rapport au gouvernement de la République française relatif à la visite effectuée par le CPT en France, du 27 octobre au 8 novembre 1991, CPT / Inf (93) 2 (FR), \$138. 
"changements spectaculaires» dans les États obtenus grâce à l'action du Comité et notamment « la suppression, dans un grand nombre de pays, des volets métalliques qui obscurcissaient en permanence les cellules, obligeant les détenus à vivre sans lumière ou sous lumière artificielle (ainsi que) les cellules trop petites ». «Les détenus peuvent aussi bénéficier plus rapidement qu'autrefois de l'assistance d'un avocat et de soins médicaux, notamment dans les commissariats de police ${ }^{48}$. Ces changements sont réels mais on ne peut probablement pas les imputer à l'action du seul Comité.

En dehors de ces exemples où l'intervention du Comité constitue indéniablement le facteur d'évolution directe ou indirecte, les effets positifs de ses actions sont souvent plus diffus, discrets et prennent place dans le temps. La vision très positive du CPT ne doit pas en effet aboutir à occulter les limites de ce contrôle, en particulier parce que le principe de confidentialité du Comité peut parfois s'apparenter à une discrétion excessive. On peut en effet estimer que le CPT, institution jeune, a pu souffrir d'un déficit d'image et que les États membres n'en avaient ou n'en ont encore qu'une connaissance parcellaire. Le CPT a d'ailleurs pris rapidement conscience de ce risque et dès son Premier rapport général d'activités, demandait lui-même «aux États parties d'informer, par des moyens appropriés (instructions, circulaires, etc.) les autorités nationales et le personnel concerné (directeurs d'établissements pénitentiaires, gardiens de prison, fonctionnaires de police, etc.) de l'existence, des pouvoirs et du rôle du Comité ${ }^{49}$. Cette visibilité du Comité semble aujourd'hui acquise et le fait que les principaux éléments des rapports rendus publics par les États fassent l'objet d'un compte rendu au sein des grands journaux constitue une mesure de publicité considérable pour lui. En 1996, la deuxième visite du Comité en France a ainsi connu un retentissement important, suite à la publication dans le journal Le Monde de larges extraits du rapport publié en 1998, concernant spécialement les conditions matérielles d'incarcération à la prison de la Santé à Paris ${ }^{50}$. A contrario, même si l'on peut déplorer le procédé, le fait qu'une agence de presse communique un rapport avant la publication officielle constitue un formidable élément de reconnaissance de l'importance du travail du Comité ${ }^{51}$.

La pratique montre cependant des éléments de résistance étatique et l'on peut percevoir assez aisément les limites de la portée de l'action du Comité. Il ne peut être qu'un pouvoir de persuasion, d'incitation. Si les États répondent le plus souvent de manière très précise et détaillée aux recommandations et réflexions du CPT, celui-ci se trouve néanmoins tout à fait démuni lorsque l'État mis en cause se contente de répondre de manière très générale en n'offrant comme seul argument que le droit en vigueur dans l'État. Ainsi, face aux critiques du CPT indiquant qu'il faut «rappeler aux fonctionnaires pénitentiaires du centre pénitentiaire de Lannemezan qu'il ne faut pas employer plus de force qu'il n'est strictement nécessaire et que, dès lors que des détenus violents et/ou récalcitrants ont été maîtrisés, rien ne saurait jamais justifier qu'ils soient brutalisés ", le gouvernement français se contente de rappeler les articles pertinents du Code de procédure pénale ${ }^{52}$. À l'évidence, le Comité ne sollicitait pas une telle leçon de droit français mais attendait bien plutôt que le gouvernement indique matériellement comment ces dispositions sont mises en œuvre dans l'établissement concerné... De même, lorsque le CPT demande au gouvernement français de «reconsidérer la prohibition générale faite aux détenus de téléphoner », la réponse très laconique est seulement que "L'accès des détenus au téléphone fait également l'objet de réflexions dans le cadre de l'élaboration de la loi pénitentiaire ${ }^{53}$. Le CPT est évidemment totalement dépourvu de moyens d'action face à des réponses d'un État qui s'apparentent assez clairement à de la mauvaise foi ${ }^{54}$. Très régulièrement les États se contentent de répondre a minima aux observations du CPT, rappelant l'état du droit en vigueur ou utilisant des formules vagues comme « des instructions seront données pour... » ou « une attitude plus compréhensive sera accordée à...» ou «la Direction concernée (de tel établissement) a assuré qu'elle mettait en œuvre tous les efforts possibles... ${ }^{55}$.

En outre, rien n'empêche aujourd'hui un État de développer des options en matière de politique carcérale

48. Interview du 26 juin 2002. source : http://www.coe.int/T/F/Com/Dossiers/Themes/Torture/InterviewCasale2.ASP

49. Premier rapport général d'activités, $\$ 91$ sq.

50. Voir également D. Saubaber, «Le Comité de prévention de la torture critique les carences des zones d'attente», Le Monde, 17 décembre 2003 (à propos de la publication du rapport du CPT suite à la visite de l'aéroport Charles de Gaulle).

51. Publication en janvier 2004 par l'Agence France Presse d'extraits du rapport d'une visite ad hoc du Comité dans trois établissements pénitentiaires français en juin 2003. Voir supra, note 13 .

52. «L'article D. 283-5 du Code de procédure pénale dispose que le personnel de l'Administration pénitentiaire ne doit utiliser la force envers les détenus qu'en cas de légitime défense, de tentative d'évasion ou de résistance par la violence ou par inertie physique aux ordres donnés. Lorsqu'il y recourt, il ne peut le faire qu'en se limitant à ce qui est strictement nécessaire. Conformément à l'article D. 220 du Code de procédure pénale, il est interdit aux agents des services déconcentrés de l'Administration pénitentiaire et aux personnes ayant accès à la détention de se livrer à des actes de violence sur les détenus, d'user à leur égard, soit de dénominations injurieuses, soit de tutoiement, soit de langage grossier ou familier ", Réponse du gouvernement de la République française au rapport du CPT relatif à sa visite effectuée en France du 14 au 26 mai 2000.

53. Ibid., $\$ 116$.

54. Comme lorsque le gouvernement italien répond à une critique du CPT dénonçant une ambiance trop «régimentée » dans une maison d'arrêt napolitaine par l'argument suivant : «L'usage observé par le CPT selon lequel, à l'occasion de leurs déplacements à l'intérieur de l'établissement, les détenus marchaient en rangs par deux les mains derrière le dos, ne répond pas à un style imposé par le personnel de police pénitentiaire désormais exempt d'approches de style militaire mais probablement à une habitude observée par les détenus qu'il est difficile d'extirper »..., Réponse du gouvernement italien au rapport du CPT relatif à sa visite effectuée en Italie du 13 au 25 février 2000, CPT / Inf (2003) 17.

55. Voir par exemple le Rapport du gouvernement du Grand-Duché de Luxembourg en réponse au rapport du CPT relatif à sa visite au Luxembourg du 20 au 25 avril 1997, CPT/Inf (98) 16 [FR] (partie II), 1998. 
qui vont à l'encontre des critères exprimés par le CPT. Celui-ci a ainsi eu l'occasion d'exprimer sa franche opposition aux politiques de construction de nouveaux établissements conçues comme solution au surpeuplement carcéral. Cette position exprimée très clairement dans le Septième rapport général d'activités ${ }^{56}$ n'a nullement empêché le nouveau gouvernement français en place depuis 2002 de s'orienter dans la voie du renforcement massif des capacités d'accueil des établissements pénitentiaires. La création au sein du gouvernement Raffarin d'un inédit secrétariat d'État aux programmes immobiliers de la justice (portefeuille confié initialement à M. Pierre Bédier, puis à $\mathrm{M}^{\mathrm{me}}$ Nicole Guedj à partir de janvier 2004) montre bien l'importance de cette politique d'ouverture de nouvelles places de détention: en novembre 2002, le gouvernement annonçait l'ouverture d'ici 2007 de 13200 nouvelles places de prison, programme d'un montant de 1,4 milliard d'euros.

Enfin, quelle est la portée véritable de «l'arme absolue » entre les mains du Comité que constitue la déclaration publique? Si l'on peut considérer que l'application de cette technique prévue par l'article 10.2 de la Convention de 1987 a permis d'amener certaines modifications dans les prisons turques, on peut aussi penser que son utilisation à l'encontre de la Russie risque de provoquer bien peu de changements en République de Tchétchénie ${ }^{57}$.

On peut en fait se demander si le succès le plus manifeste du CPT ne réside pas dans son existence même et dans le type de contrôle qu'il effectue. Au-delà même des recommandations présentées par le Comité, c'est sa structure même qui apparaît de plus en plus souvent comme un modèle à suivre. En effet, le Comité semble aujourd'hui constituer un véritable standard de contrôle de haut niveau, que certains États souhaiteraient voir transposé au plan interne. Ainsi, dans ces conclusions, le premier président de la Cour de cassation, M. Guy Canivet, propose l'instauration d'une structure de "vérification », mission confiée à une autorité dénommée «Contrôleur général des prisons », assistée d'un corps de contrôleurs permanents, de collaborateurs occasionnels, et doté de services administratifs. Cette structure présente des analogies extrêmement marquées avec le CPT, tant en ce qui concerne son statut que ses missions. La description des visites qui relèveraient de cette institution nouvelle laisse facilement percevoir quelle a été la source d'inspiration. Le rapport préconise en effet des « visites programmées » (vérification complète de chaque établissement selon une périodicité fixée), des «visites inopinées » (déclenchées par la communication d'une information, la réception d'une plainte ou d'une dénonciation) et enfin la « visite de suivi » (destinée à assurer la continuité dans le contrôle), qui ressemblent assez fortement aux visites organisées par le CPT. Plus précisément encore, la proposition de loi pénitentiaire déposée au Sénat par MM. Hyest et Cabanel en mai 2001 constitue un autre indice important de l'influence véritable du Comité sur la politique pénitentiaire française. Pour la ministre de la Justice de l'époque, $\mathrm{M}^{\mathrm{me}}$ Lebranchu, commentant les dispositions de la proposition instaurant un contrôle général des prisons, « Le principe même d'un contrôle extérieur des établissements pénitentiaires ne paraît pas discutable». Ce contrôle permet en effet de vérifier les conditions concrètes de la privation de liberté, mais également de protéger les personnels « parfois confrontés à des accusations injustes que l'administration peut moins aisément dissiper qu'une autorité indépendante, car le soupçon de collusion, bien qu'injustifié, est tenace ». Pour la ministre, il était en effet possible de concevoir un système portant plus spécifiquement sur les conditions dans lesquelles est assuré le respect des droits des détenus, en particulier les droits à la dignité et à la sécurité. Or, cela « renvoie à un dispositif que nous connaissons bien et qui est celui du Comité pour la prévention de la torture et des traitements inhumains et dégradants ${ }^{58}$.

En apparaissant comme une institution de référence, le CPT provoque et suggère réformes et modifications au sein des États membres. Mais cette influence est également fortement visible au plan international, tant le Comité constitue désormais une source d'inspiration première pour instaurer des mécanismes de protection internationale contre la torture.

\section{B. Une source d'inspiration pour la protection internationale contre la torture et les peines ou traitements inhumains et dégradants}

La Convention européenne de 1987 a toujours entretenu des liens particuliers avec l'échelon international en particulier parce que le CPT a pris l'habitude d'établir des relations de travail non seulement avec la Cour européenne des droits de l'homme, mais également avec le Comité international de la Croix-Rouge (CICR), le Comité des Nations Unies contre la torture, le Rapporteur spécial des Nations Unies sur les questions se rapportant à la torture, le Haut Commissariat des Nations Unies pour les réfugiés, et même avec plusieurs organisations non gouvernementales. En outre, les sources intellectuelles des textes européen et onusien sont largement communes. C'est en effet une proposition formulée en

56. «Certains pays ont pris pour option d'accroître leur parc pénitentiaire. Pour sa part, le CPT est loin d'être convaincu que l'accroissement des capacités d'accueil constituera à lui seul une solution durable. En effet, plusieurs États européens se sont lancés dans de vastes programmes de construction d'établissements pénitentiaires pour découvrir que leur population carcérale augmentait de concert. À l'inverse, dans certains États, l'existence de politiques visant à limiter ou moduler le nombre de personnes emprisonnées a contribué de manière importante au maintien de la population carcérale à un niveau gérable », 1997, CPT / Inf (97) 10 (FR), \$14.

57. Dans sa déclaration publique du 10 juillet 2003 (doc. CPT / Inf (2003) 33), le Comité mentionne d'ailleurs explicitement que « en dépit d'efforts soutenus de la part du CPT au cours des deux dernières années, les autorités russes n'ont pas traité de façon efficace certains problèmes majeurs liés au mandat du Comité» $(\$ 3)$.

58. Discours de $\mathrm{M}^{\text {me }}$ Marylise Lebranchu, Ministre de la Justice, Garde des Sceaux, source: http://www.justice.gouv.fr/discours/d260401.htm 
1976 par un banquier helvétique, Jean-Jacques Gautier, qui va conduire à une adoption presque parallèle de deux textes, l'un international, l'autre régional. Dans un rapport élaboré à la demande du gouvernement suisse, JeanJacques Gautier, président du Comité suisse contre la torture, proposait l'élaboration d'une Convention, instaurant un système de visites de tous les lieux de détention par des experts indépendants. L'inspiration directe est évidemment celle du Comité international de la CroixRouge. Mais le champ d'application du texte proposé est plus vaste puisqu'il doit s'étendre à tous les lieux où des personnes sont privées de leur liberté.

Or, dès 1973, les Nations Unies avaient commencé à réfléchir à « la question des droits de l'homme dans le cas des personnes soumises à toute forme de détention ou d'emprisonnement ${ }^{59}$, mais c'est en 1977 que l'Assemblée générale des Nations Unies va charger la Commission des droits de l'homme d'élaborer un projet de convention contre la torture, poursuivant le travail entamé par la résolution du 9 décembre $1975^{60}$. Ces travaux aboutissent à l'adoption par l'Assemblée générale le 10 décembre 1984 de la Convention contre la torture et autres peines ou traitements inhumains et dégradants. Les fractures multiples qui se développeront entre les représentants étatiques lors de l'élaboration du texte se cristalliseront autour d'un point, qui va constituer le maillon faible de ce nouveau texte conventionnel: malgré les efforts de plusieurs États (et notamment la présentation par le Costa Rica, le 6 mars 1980, d'un projet de Protocole facultatif à la Convention), le mécanisme de contrôle du texte reste assez timide et en tout cas peu original par rapport aux «canons" habituels de contrôle des textes internationaux. C'est en effet par la voie d'un Comité de dix experts indépendant (le «Comité contre la torture») que ce texte est protégé. Ce Comité est essentiellement habilité à examiner les rapports transmis périodiquement par les États afin de présenter les mesures prises pour donner effet à leurs engagements ${ }^{61}$. Le Comité peut également recevoir des communications émanant d'un État ou d'un particulier, mais il s'agit là d'un simple protocole facultatif ${ }^{62}$. L'organisation mise en place au plan onusien s'est donc révélée particulièrement en retrait par rapport à la volonté de M. Gautier.
La défaillance du système élaboré au plan international explique très largement que ce soit dans le cadre du Conseil de l'Europe que l'on va s'attacher à donner à cet ambitieux projet une force juridique. En janvier 1981, la Recommandation 909 (1981) adoptée par l'Assemblée parlementaire du Conseil de l'Europe se référait aux travaux entrepris dans le cadre des Nations Unies et recommandait au Comité des ministres d'inviter les gouvernements des États membres à hâter l'adoption et la mise en vigueur du projet de convention contre la torture élaboré par la Commission des droits de l'homme des Nations Unies. Elle invitait également les gouvernements des États membres représentés à cette commission à faire tout leur possible pour que cette dernière examine avec une attention particulière le projet de protocole facultatif à la Convention (présenté par le Costa Rica), dès que le projet de convention aurait lui-même été soumis au Conseil économique et social des Nations Unies. Les travaux d'élaboration d'un texte prohibant la torture et les traitements inhumains et dégradants se sont donc poursuivis de manière parallèle dans le cadre onusien et européen. C'est peut-être le président de la commission juridique de l'Assemblée parlementaire du Conseil de l'Europe, M. Berrier, qui va le mieux définir la mission d'influence que doit jouer la Convention européenne en se demandant en 1983 «[...] si les pays européens ne pourraient pas donner l'exemple et établir un tel système entre eux dans le cadre du Conseil de l'Europe " ${ }^{63}$. Dès le départ, le système élaboré dans le cadre continental est analysé comme une source d'influence majeure pour le plan international, un " précédent » digne de valeur, «avec, en arrière-plan, l'idée de l'application de ses règles hors des États membres du Conseil de l'Europe " ${ }^{64}$.

À partir de 1987 existent donc deux textes comparables ${ }^{65}$, à l'objet identique, puisqu'il s'agit de la prohibition de la torture et des peines ou traitements inhumains ou dégradants, mais mettant en œuvre des techniques et principes différents: le texte onusien se fonde sur une définition explicite des actes prohibés mais instaure un mode de contrôle et de surveillance très timide. En revanche, la Convention européenne de 1987 se concentre sur le seul dispositif de protection qu'est le Comité européen pour la prévention de la torture.

59. Demande formulée par la Commission des droits de l'homme à sa Sous-Commission de lutte contre les mesures discriminatoires et de la protection des minorités. Voir Z. Haquani, «La Convention de l'ONU contre la torture », Revue générale de droit international public, 1986, p. 135.

60. Déclaration sur la protection de toutes les personnes contre la torture et autres peines ou traitements cruels, inhumains ou dégradants, adoptée par l'Assemblée générale des Nations Unies le 9 décembre 1975, résolution 3452 (XXX).

61. La liste des États en retard pour la transmission de leurs rapports étant particulièrement impressionnante: en 2002, le Comité relevait 119 rapports qui n'avaient toujours pas été transmis, certains depuis 14 ans! (la France a, pour sa part, omis de transmettre ses troisième et quatrième rapports périodiques pourtant prévus respectivement pour 1996 et 2000). Voir Rapport du Comité contre la torture pour les $37^{\mathrm{e}}$ et $38^{\mathrm{e}}$ sessions (2001-2002), doc A $57 / 44$.

62. Protocole facultatif qui n'a été ratifié que par 55 États (le total d'États parties à la Convention s'élevant à 132 au 19 janvier 2004 ). D'où un système de requêtes qui n'a abouti qu'au dépôt de 242 plaintes, la majorité d'entre elles visant la Suède (52), la Suisse (44), le Canada (36), la France (29)... On peut difficilement imaginer qu'il s'agisse là de l'image fidèle des cas de torture existant dans le monde! source: http://www.unhchr.ch/html/menu2/ 8/stat3.htm

63. AS / Jur $((33) 18, \$ 13$.

64. A. Cassese, Inhuman States..., p. 13 .

65. Sans parler des autres éléments de protection mis en place dans le cadre onusien. Ainsi depuis 1986, les Nations Unies ont décidé d’instaurer un Rapporteur spécial concernant les questions relatives à la torture. Doté d'un large mandat, il est chargé d'agir sur les questions relatives à la torture survenant partout dans le monde. À la différence du Comité, son mandat n'est pas limité aux États parties à la Convention contre la torture mais s'étend à tous les États. 
Plusieurs signes montrent que le texte européen constitue une source d'inspiration directe pour l'évolution de son homologue international. Ainsi, lorsque le 6 mars 1989, la Commission des droits de l'homme des Nations Unies inscrit à nouveau à son ordre du jour le projet de protocole facultatif costaricain, la Commission exprime l'avis selon lequel avant d'examiner ce texte, il serait opportun «de prendre note... de l'expérience de la Convention européenne pour la prévention de la torture » (ce qui conduira à décider d'un report de l'examen du texte costaricain à la $47^{\mathrm{e}}$ session de la Commission en 1991). Ce Protocole facultatif finit par être adopté par l'Assemblée générale des Nations Unies le 18 décembre 2002. Ouvert à la signature le $1^{\text {er }}$ janvier 2003, il entrera en vigueur à la $20^{\mathrm{e}}$ ratification ${ }^{66}$. Les 127 États qui ont adopté ce texte ${ }^{67}$ mettent en place un nouvel organe international (le Sous-Comité du Comité contre la torture) et imposent aux États parties de se doter de mécanismes nationaux complémentaires de prévention contre la torture. La mission de ce Sous-Comité s'inspire très fortement du $\mathrm{CPT}$, puisque la nouvelle structure internationale sera également chargée d'effectuer des visites des lieux de détention afin de prévenir la torture et d'améliorer les conditions de détention. Plus précisément encore, le Sous-Comité entreprendra des visites périodiques dans tous les États parties, en maintenant un dialogue avec à la fois l'État partie en question et l'organe national de visite ${ }^{68}$. Ces visites, simplement notifiées à l'État concerné, se dérouleront de manière confidentielle, avec rencontre des autorités locales, entretiens sans témoin avec des détenus, contacts avec diverses sources d'informations (dont les organisations non gouvernementales). Un rapport fera état des conditions de détention constatées et émettra des recommandations concrètes, sur la base desquelles un dialogue s'instaurera avec l'État. Si celui-ci rechigne à toute coopération et amélioration, le SousComité sera enclin à solliciter de la part du Comité contre la torture une déclaration publique ou la publication du rapport. Les États parties seront obligés de coopérer avec les mécanismes de visite et de prendre les mesures qui s'imposent pour mettre en œuvre leurs recommandations. De plus, ces États se verront dans l'obligation de garantir l'indépendance fonctionnelle des organes nationaux de visite, de s'assurer que leurs membres ont les capacités et compétences professionnelles requises et de mettre à leur disposition les ressources nécessaires pour mener à bien leur mission. Le travail mené par le CPT a été sans nul doute déterminant, en démontrant que les mécanismes de visite peuvent revêtir un rôle de prévention important, pour l'amélioration des conditions de détention et la prévention de la torture et des traitements inhumains ou dégradants. En raison de la proximité des deux textes, on ne peut que comprendre que le CPT se félicite « de cette évolution et se réjouit de coopérer avec le Sous-Comité de la prévention de la torture qui sera créé dans le cadre du Protocole facultatif et, dans les pays qui auront ratifié à la fois la Convention et le Protocole facultatif, avec les mécanismes nationaux de prévention prévus par le Protocole ${ }^{69}$.

Le CPT, par sa fonction " pionnière ${ }^{70}$, se situe bien dans la perspective générale d'un Conseil de l'Europe chargé de promouvoir les idéaux démocratiques et de favoriser les conditions de développement de l'État de droit et des libertés fondamentales au sein des États membres. Grâce à la qualité de son travail, le CPT permet, si ce n'est une entrée, du moins un regard porté par la société civile dans les établissements pénitentiaires. Cette volonté de rendre le monde pénitentiaire plus lisible et plus transparent, permet de ne pas oublier que les prisons constituent une partie intégrante de la société. Bien plus encore, ces prisons constituent un puissant révélateur de l'état de chaque société. En révélant inlassablement les lacunes, dysfonctionnements, mais aussi progrès des établissements où l'on prive des individus de leur liberté, le CPT, à son échelle, contribue à conforter l'analyse selon laquelle l'état d'une démocratie se vérifie aussi dans ses prisons.

66. Au $1^{\text {er }}$ janvier 2004, le Protocole a fait l'objet de 3 ratifications et 20 signatures.

67. 42 se sont abstenus et 4 ont voté contre (États-Unis, Nigéria, Îles Marshall et Palau). Le Protocole a été soutenu par une majorité multirégionale d’États, menée par le Costa Rica et la Suisse.

68. On peut même estimer que le Protocole facultatif va plus loin que le texte européen puisque selon les articles 3 et 17, les États parties sont tenus de mettre en place, un an après l'entrée en vigueur du Protocole facultatif ou, si ce dernier est déjà entré en vigueur, un an après l'avoir ratifié, de désigner et d'administrer un ou plusieurs mécanismes nationaux indépendants chargés de visiter les lieux de détention. L’idée est ici que ces mécanismes nationaux seront capables d'entreprendre des visites beaucoup plus approfondies que le Sous-Comité, car bénéficiant d'une meilleure connaissance de la situation locale et d'un potentiel plus important de suivi. Les organes internationaux et nationaux devront néanmoins travailler ensemble pour organiser des visites préventives et formuler des recommandations aux autorités.

69. Treizième rapport général d'activités, 2003, \$21, doc CPT / Inf (2003) 35. Cette existence d'un double système pouvant toutefois poser quelques problèmes de coordination le CPT recommande d'adopter une proposition selon laquelle les Parties à la Convention européenne de 1987 qui ratifient aussi le Protocole facultatif pourraient accepter que les rapports sur les visites effectuées par le CPT dans leurs pays et leurs réponses soient systématiquement transmis au Sous-Comité à titre confidentiel. Ainsi, les consultations entre le Sous-Comité et le CPT auraient lieu à la lumière de tous les faits pertinents $(\$ 22)$.

70. Selon les termes employés par G. Kaiser, «Detention in Europe and the European Committee for the Prevention of Torture », European Journal of Crime, Criminal law and Criminal Justice, vol. 3, 1995, p. 15 sq. 
